Hans Mathias

Kepplinger

\title{
(Ab)Bilder - wie Medien Realität produzieren
}

Vier Punkte will ich ansprechen: erstens die Verfälschung von Bildern, d.h. die Verfälschung von Darstellungen; zweitens die Verfälschung des Dargestellten, d.h. dessen, was die Bilder abzubilden scheinen; drittens die Verfälschung des Dargestellten durch die Bildauswahl, und viertens die Irreführung durch den emotionalisierenden Charakter von Bildern. Danach werde ich einige Folgerungen ziehen. Sie werden sich vielleicht fragen: Warum immer nur Verfälschung, warum immer nur das Negative? Natürlich gibt es viele Bilder, die die Realität so darstellen, dass man sich realitätsgerecht daran orientieren kann. Aber das ist nicht problematisch, und insofern wähle ich einen etwas anderen Zugriff auf die Thematik.

\section{Erstens: Die Verfälschung der Darstellung}

In Berlin läuft im Moment eine Ausstellung mit manipulierten Fotos. Unter anderem sieht man eine Aufnahme von Lenin bei einer Rede in Moskau am 5. Mai 1920. Auf dem Foto ist auch Leo Trotzki abgebildet, der später wegretuschiert und durch Holzstufen ersetzt wurde, weil er inzwischen in Ungnade gefallen war. Man sieht auch ein Agenturfoto von Helmut Kohl mit Bill Clinton und Bernard Vogel in Eisenach vor einer eindruckvollen Menschenmenge. Die Originalaufnahme zeigt in der Menschenmenge ein Plakat mit der Aufschrift "Ihr habt auch in schlechten Zeiten dicke Backen“, das für eine spätere Publikation wegretuschiert worden war. Ein anderes Beispiel, an das Sie sich vielleicht noch erinnern, ist eine Aufnahme von Lady Di mit ihrem Geliebten Dodi auf einer Yacht im Mittelmeer. Es sieht so aus, als ob sie sich gerade küssen wollten. In Wirklichkeit saßen beide relativ weit auseinander, aber die Redaktion einer englischen Tageszeitung hatte den Raum zwischen ihnen teilweise weggeschnitten, so dass der erwähnte Eindruck entstand.

Die Möglichkeiten zur Verfälschung von Bildern haben in den letzten Jahren durch die digitale Bildbearbeitung sehr zugenommen. Es gibt in den Illustrierten kaum noch Stars, die unangenehme Muttermale oder Ähnliches haben. Sie werden wegretuschiert. Es werden Busen akzentuiert, die nicht vorhanden sind, und es werden vorhandene Hintergründe weggenommen oder fehlende hinzugefügt. Es werden Personen wegretuschiert, zusammengerückt oder eingefügt usw. Uns empört das. Es wird mit Recht kritisiert, und die Kritik erscheint uns normal. Aber trotzdem 
stellt sich die Frage: Warum empören wir uns darüber? Es gibt zwei Gründe: Wir fühlen uns erstens verletzt, weil wir uns getäuscht fühlen. Wir fühlen uns hinters Licht geführt, und das ärgert uns. Und wir kritisieren zweitens solche Manipulationen, weil zuweilen die Persönlichkeitsrechte der Abgebildeten verletzt werden, also z.B. von Lady Di. Hier spielen presserechtliche Aspekte hinein.

Lassen Sie uns das Problem einen Schritt weiter führen. Was steckt hinter unserem Ärger? Dahinter steckt letztlich das Vertrauen, dass Fotografien, wenn sie nicht manipuliert sind, die Wirklichkeit so darstellen, wie sie ist. Das heißt: Eine nicht manipulativ veränderte Fotografie stellt die Wirklichkeit unverfälscht dar. Erst die Veränderung der Fotografie führt zu einer verfälschten Darstellung von Wirklichkeit. Folglich können wir die Realität anhand dieser Bilder nicht mehr erkennen. Damit stellt sich die Frage: Stimmt die Prämisse? Stellen die Fotos die Wirklichkeit tatsächlich so dar, wie sie ist? Sind die Menschen, die auf Bildern dargestellt werden, wirklich so, wie sie da erscheinen? Das führt mich zum zweiten Punkt, nämlich zur Verfälschung des Dargestellten, also zur Verfälschung dessen, was dargestellt wird.

\section{Zweitens: Die Verfälschung des Dargestellten}

Das auf Fotos Dargestellte kann auf mehrere Weisen verfälscht sein. Die Wirklichkeit, die dargestellt wird, kann schon durch situationsbedingte Verhaltensänderungen der Personen, die abgebildet werden, verfälscht sein. Wenn Sie sich eine Fernseh-Reportage über einen Supermarkt vorstellen: Sie sehen Verkäuferinnen an der Kasse, die alle frisch frisiert sind und schöne Kleider anhaben, sozusagen im Sonntagsstaat sind. Natürlich sitzen die normalerweise nicht so da, aber sie wissen, es kommt das Fernsehen, also machen sie sich fein. Das kann man systematisch untersuchen. In Rheinland-Pfalz gibt es eine Sendereihe, für die ein Radio-Team über kleine Dörfer reist und die Bewohner interviewt. Wir hatten die Vermutung, dass sie sich, wenn die Radioleute kommen, anders verhalten als normalerweise. Also haben wir eine Studentin für ihre Examensarbeit einige Tage vorher in die Dörfer geschickt. Sie hat in Protokollen festgehalten, wie sich die Menschen normalerweise z.B. im Wirtshaus benehmen - wer mit wem spricht, welche Kleidung die Menschen anhaben usw.

Zwischen dem normalen Verhalten und dem Verhalten in Anwesenheit der Journalisten liegen Welten. Ein Beispiel: Wenn die Radioleute da sind, sprechen sich Honoratioren oft mit Titel an: „Wie der Herr Bürgermeister schon gesagt hat...", was den Gesprächen einen geradezu 
würdevollen Charakter verleiht. Das machen die Dorfbewohner normalerweise natürlich nie, weil jeder weiß, dass der - sagen wir mal - Fritz Müller der Bürgermeister ist. Wenn die Radioleute da sind, unterstellen sie aber zu Recht, dass die Hörer das nicht wissen. Also muss man Fritz Müller als Bürgermeister ansprechen. Diese Veränderung der Situation schlägt sich auch in der Art und Weise nieder, wie gesprochen wird. Die Dorfbewohner sprechen dann keinen normalen Dialekt mehr, sondern ein aufgesetztes Hochdeutsch. Und selbstverständlich kann man ähnliche Veränderungen in der Kleidung beobachten, in der Gestik, in der Mimik usw. Daraus folgt, dass die Medien die Wirklichkeit schon deshalb nicht so darstellen, wie sie normalerweise ist, weil diejenigen, über die berichtet wird, im Angesicht der Aufnahmegeräte für den Hörfunk und das Fernsehen ihr Verhalten ändern. Nichts ist so wenig authentisch wie die aktuelle Berichterstattung des Fernsehens.

Die Wirkung der Medien auf diejenigen, über die sie berichten, bezeichnet man als „reziproke Effekte“. Sie wurden zuerst 1953 bei der Rückkehr von General McArthur aus Korea bemerkt. McArthur, der große Held des Pazifik-Krieges, war von Präsident Eisenhower aus Korea abberufen worden, weil er gegen den erklärten Willen von Eisenhower den Einsatz von Atombomben gefordert hatte. McArthur kam auf seiner Rückreise in Seattle an, reiste vom Westen nach Osten durch die USA, und das damals noch neue Fernsehen verbreitete - zu Recht oder Unrecht, das sei dahingestellt - den Eindruck, dass die Menschen dem General zujubelten. Als McArthur nach Chicago kam, wollten die beiden Studenten Kurt Lang und Gladys Engel Lang untersuchen, warum sich die Massen so euphorisch verhielten. Dazu hatten sie zahlreiche Beobachter an der Fahrtstrecke von General McArthur postiert, die alle Einzelheiten auf Beobachtungsbögen protokollierten. Weil Gladys Engel Lang schwanger war, konnte sie selbst nicht mit hinausgehen. Deshalb protokollierte sie das Geschehen mit einigen Freunden anhand der Fernsehberichterstattung. Zur Überraschung der beiden Langs stimmten die Beobachtungen anhand der Fernsehberichterstattung nicht mit den Beobachtungen auf der Straße überein: Auf den Straßen gab es nur ganz kleine Gruppen, im Fernsehen sahen sie wie große Menschenmengen aus, weil sie von den Fernsehleuten geschickt aufgenommen worden waren. Vor Ort gab es kaum Jubel für McArthur. Im Fernsehen erschien es aber so, weil die Menschen oft in die Kameras gewunken haben. So entstand im Fernsehen der Eindruck von jubelnden Menschenmengen, die in Wirklichkeit gar nicht existierten.

Reziproke EIfekte besitzen eine große praktische Relevanz für das politische Leben. Es gibt heute - vereinfacht gesagt - zwei Typen von Politikern: Zum einen die Fernsehbegabungen - „Rotlichtenthemmte“, 
die geradezu aufblühen, wenn das Rotlicht an der Kamera angeht, die gerade auf Sendung ist. Sie sind innerhalb von Sekunden in der Lage, knappe, witzige und überzeugend klingende Bemerkung̈en zu machen. Sie sind sofort „auf Sendung“. Dazu gehören u. a. Gerhard Schröder, Gregor Gysi, Joschka Fischer und Guido Westerwelle. Auf der anderen Seite gibt es Fernsehlegastheniker, „Rotlichtgehemmte“, die dazu sehr viel weniger in der Lage sind. Dazu gehören u a. Helmut Kohl, Angela Merkel, Edmund Stoiber, Klaus Kinkel, Olaf Scholz und Wolfgang Gerhardt. Das sind Personen, die nicht in gleicher Weise innerhalb von 10 , 15,30 Sekunden etwas rüberbringen, was den Leuten überzeugend erscheint. Das bedeutet nicht, dass sie nichts zu sagen hätten oder dass sie nicht sagen könnten, was sie zu sagen haben. Es bedeutet nur, dass sie es nicht so mitteilen können, dass es im Fernsehen spontan einen positiven Eindruck macht.

Die unterschiedliche Fernsehbegabung von Politikern hat angesichts der Bedeutung des Fernsehens als Fenster zur Wahlbevölkerung Rückwirkungen auf die Politik. Wenn Sie sich die deutsche Parteienlandschaft ansehen, werden Sie feststellen, dass fast alle Führungsfiguren herausragende Fernsehbegabungen sind. An der Spitze der SPD steht Gerhard Schröder statt Oskar Lafontaine. Obwohl die Parteibasis Lafontaine wollte, wurde Schröder im Frühjahr 1998 vor allem deshalb zum Kanzlerkandidaten der SPD ernannt, weil er die größeren Chancen bei den Medien hat. An der FDP-Spitze steht Guido Westerwelle und nicht mehr Wolfgang Gerhardt. Man kann streiten, wer in der Sache der bessere liberale Politiker ist. Aber im Fernsehen ist Westerwelle zweifellos der Erfolgreichere. Bei den Grünen hat Joschka Fischer nie ein bedeutendes Amt innegehabt. Aber er war immer ihr bedeutendster Politiker, vor allem deshalb, weil er ein begnadeter Selbstdarsteller ist. Gregor Gysi ist in der PDS ein ähnlicher Fall.

Im mediendominierten Ausland ist es ähnlich: Tony Blair ist eine eminente Fernsehbegabung, und das Hauptproblem der Konservativen besteht darin, dass sie niemanden haben, der ihm dort das Wasser reichen kann. Man muss sehen, mit welcher Souveränität er auftritt, wenn er unter extremem Druck steht - wie in den Wochen und Monaten nach dem Ende des Irak-Kriegs und während der ergebnislosen Suche nach dem angeblichen Kriegsgrund, den Massenvernichtungswaffen. Das kann man nicht lernen. Ein anderes Beispiel: Nehmen Sie Ronald Reagan oder als Parodie Arnold Schwarzenegger. Wir erleben das Vordringen von Starpolitikern oder Polit-Stars. Damit bezeichne ich Leute, die ihre Karriere nicht in der Partei von unten nach oben machen, sondern Prominenz über die Medien - vor allem das Fernsehen - erwerben und diese Prominenz als Seiteneinsteiger oben in die Partei einbringen. Die über 
das Fernsehen erworbene Prominenz wird als Machtbasis in die Partei eingebracht und von der Basis nur noch abgenickt. Sie wird vor die Frage gestellt: Wollt ihr diesen Hoffnungsträger, oder wollt ihr ihn nicht? Das ändert Politik, das ändert die Karrierebedingungen von Politikern, und das ändert natürlich auch den Inhalt und die Art, wie Politik gemacht wird.

Ich habe gesagt, ein Grund, weshalb die Medien - vor allem das Fernsehen - die Wirklichkeit nicht so darstellen, wie sie ist, sind die Verhaltensänderungen der Dargestellten. Der zweite Grund besteht natürlich in den Verhaltensweisen der Darsteller, der Berichterstatter. Sie korrigieren, sie manipulieren - nehmen Sie das Wort nicht zu negativ - die Realität zum Zwecke der Darstellung. Das beginnt ganz harmlos etwa damit, dass bei Gruppenaufnahmen die Menschen anders arrangiert werden als sie sich normalerweise hinstellen. Dann stehen nicht die befreundeten Politiker zusammen, sondern die aus Sicht der Berichterstatter wichtigen. Aber dabei bleibt es nicht. So berichtet der Journalist Udo Ulfkotte, dass er auf mehreren Kriegsschauplätzen beobachtet hat, wie Fernsehteams ausgebrannte Panzer mit Benzin, das sie eigens dabeihatten, wieder in Brand gesteckt haben, um sie als Hintergrund für journalistische Analysen aus dem Kriegsgebiet zu filmen.

Als Zwischenbilanz kann man festhalten: Die Bilder stellen die Realität oft nicht so dar, wie sie normalerweise ist, sondern wie sie wegen der Aufnahme geworden ist, aber ohne sie nie gewesen wäre. Warum die Realität im Moment der Aufnahme so ist, kann der Zuschauer in der Regel nicht erkennen. In den meisten Fällen ist das unproblematisch, weil seine Vorstellung von Realität nicht verfälscht wird. In manchen Fällen tritt aber genau das ein. Das Problem für den Zuschauer besteht darin, dass er nicht unterscheiden kann, ob seine Vorstellung irregeführt wird oder nicht. Er ist, ob er will oder nicht, der Darstellung ausgeliefert oder er traut keiner Darstellung mehr, was kaum durchzuhalten ist oder in reinem Zynismus endet.

\section{Drittens: Die Verfälschung der Darstellung durch die Selektion der Bilder}

Man kann nicht alles abbilden, und man kann nicht alle Abbildungen veröffentlichen. Selektion ist unvermeidlich. Vor dem Selektionsproblem steht der Print-Journalismus, mehr noch aber der Bild-Journalismus. Jede Selektion ist auch von der Sichtweise derjenigen geprägt, die sie vornehmen. Eir Beispiel liefert der Vietnam-Krieg. Zwei berühmte amerikanische Fotografen, David Douglas Duncan und Larry Burrows, haben mit ihren Fotografien ganz verschiedene Versionen dieses Krieges 
präsentiert. Duncan stellte auf fast vier Fünfteln seiner Aufnahmen das Kampfgeschehen dar, aber er zeigte fast nie verwundete oder tote NichtAmerikaner. Burrows stellte auf jedem zweiten Bild Zivilisten dar und konzentrierte sich auf die Verwundeten und Toten. Dabei bevorzugte er Nahaufnahmen, die Duncan fast immer vermied. Die Fotos beider zeigen die Wirklichkeit des Krieges und vermitteln doch verschiedene Eindrücke, die sehr unterschiedliche emotionale Reaktionen und gedankliche Folgerungen nahe legen. Dies war bei der Berichterstattung über den letzten Irak-Krieg nicht anders.

Zur Problematik der Bildauswahl kommt das Problem der Einordnung des Gezeigten in einen größeren Kontext. Die Betrachter von Kampfszenen oder Opfern in der Fernsehberichterstattung oder anhand von Pressefotos können in der Regel das Geschehen nicht einordnen. Sie wissen nicht, wo es stattgefunden hat, ob es typisch oder atypisch ist, wichtig oder unwichtig usw. Ein Krieg ist aber nicht die Summe der Kämpfe und nicht einmal die Summe der Schlachten. Ein Krieg ist ein extrem komplexes Geschehen, und wenn irgendwo irgendjemand einen Panzer abschießst oder ein Flugzeug oder drei Flugzeuge oder fünf, dann können Außenstehende, so eindrucksvoll die Bilder auch sein mögen, nicht abschätzen, was das für den Krieg bedeutet. Ist es kriegsentscheidend oder nebensächlich? Wir haben - auch wenn wir uns das beim Betrachten der Bilder nicht bewusst machen - keine Ahnung.

Das Fernsehen, dessen Kriegsberichterstattung besonders eindruckvoll erscheint, ist in Wirklichkeit gegenüber den Printmedien sehr benachteiligt. Wenn Sie rückblickend die Berichterstattung der „Süddeutschen Zeitung“, der „Frankfurter Allgemeine Zeitung“ oder der „Welt“ mit der Fernsehberichterstattung über den Krieg im Irak vergleichen, werden Sie feststellen: Das Fernsehen war - obwohl die aktuelle Berichterstattung des Gegenteil suggeriert hat - nicht in der Lage, den Krieg sachgerecht darzustellen, weil Fernsehberichte kaum Strukturinformationen liefern. Das Fernsehen kann Opfer zeigen, realitätsgetreu; es kann auch Kampfhandlungen zeigen, realitätsgetreu und damit auch starke Emotionen wecken - Mitleid, Entsetzen, Empörung, Hass usw. Das kann eine Zeitung nicht in gleicher Weise. Eine Zeitung kann aber durch Grafiken - was im Fernsehen möglich wäre, aber kaum genutzt wird - zeigen, wie die Geschehnisse zusammenhängen. Sie kann illustrieren, welche Bedeutung den einzelnen Ereignissen zukommt, sie kann Gewinne und Verluste in Form von Tabellen und Schaubildern präsentieren, die einem interessierten Leser wichtige Hintergrundinformationen liefern, die im Fernsehen aber kaum jemand verstehen und behalten würde. Deshalb waren und sind die Zeitungen vor allem in ihrer Berichterstattung über Kriege bei weitem bessere Quellen als die Fernsehsender. 
Durch die Selektion sind große Verzerrungsmöglichkeiten gegeben. Das kann man besonders schön an Personenfotos zeigen. Stellen die Bilder von Personen des öffentlichen Lebens diese Menschen so dar, wie sie wirklich sind? Oft ist das der Fall. Aber ist es immer der Fall? Kann man von den publizierten Fotos mit einiger Sicherheit auf den Menschen schließen, der abgebildet ist? Kann man an den Fotos den Charakter erkennen? Wir haben drei Personen innerhalb von 60 Minuten bei ihrer Berufstätigkeit so oft fotografiert, wie es möglich war. Alle Bilder zeigen nur den Kopf und die Schultern. Man sieht also nicht, was die Abgebildeten mit ihren Händen machen. Am Ende hatten wir von jeder Person etwa 120 Bilder. Ich will eine herausgreifen, die wir Horst Berger genannt haben. Im wirklichen Leben heißt er anders. Er ist Friseur in Offenbach bei Frankfurt.

Kann man aus 120 Bildern zwei Serien von Fotos machen, die völlig verschiedene Menschen zeigen? Die Antwort lautet: Man kann immer zwei solcher Serien machen, von manchen Menschen sogar drei. Eine Serie von Fotos zeigt beispielsweise Horst Berger - er war damals ein gut aussehender, grauhaariger Mann von etwa 50 Jahren - als verschmitztfreundlich, als einen nach innen lachenden, warmherzigen Menschen. Eine andere Serie zeigt ihn als einen herrischen und aggressiven, einen verbiesterten Menschen. Beide Serien bestehen aus jeweils sechs Fotos, und wir haben zwei Versuchsgruppen sieben Wochen lang jeweils Bilder aus der einen oder der anderen Serie gezeigt. Eine Gruppe bekam also nur Bilder aus der positiven Serie, die andere nur Aufnahmen aus der negativen. Wie wirkt das? Vermitteln solche Bilder nur flüchtige Eindrücke, die schnell vergessen werden? Worin bestehen die Effekte, die die Bilder auslösen? Beschränken sie sich auf die Wahrnehmung der gezeigten Person oder reichen sie darüber hinaus?

Während die Versuchspersonen Woche für Woche ein Bild aus den jeweiligen Serien sahen, haben wir ermittelt, wie sie die Person wahrnahmen, welchen Charakter sie erkannten. In der fünften Woche bekamen sie nur kurz ein neutrales Erinnerungsbild. Anschließend sollten sie aus der Erinnerung die Person beschreiben. $\mathrm{Zu}$ unserer Überraschung beschrieben die Versuchpersonen, die die unterschiedlichen Bilder gesehen hatten, Horst Berger auch eine Woche danach noch deutlich verschieden. Wir hatten in den Köpfen der Versuchspersonen sehr verschiedene Personenvorstellungen etabliert.

Bleiben die visuell vermittelten Eindrücke auf das beschränkt, was man sehen kann, oder werden sie verallgemeinert? Erstrecken sie sich also auch auf Eigenschaften und Verhaltensweisen der Personen, die man nicht sehen kann? Wir haben diesen Horst Berger in unserem Experiment als Architekten vorgestellt, weil es vom Typ her glaubhaft zu sein 
schien. Jede Woche haben wir zu den Bildern eine kurze Geschichte mit Horst Berger präsentiert und dann gefragt: Wie wird er sich verhalten, bzw. was hat er gemeint? Eine dieser Geschichten war zủm Beispiel: $\mathrm{Zu}$ Horst Berger kommt ein Bauwilliger und fragt am Ende des Gesprächs besorgt, ob die Preise stabil bleiben. Darauf sagt er: „Machen Sie sich da mal keine Sorgen“. Unsere Frage an die Versuchspersonen lautete: „Was hat Horst Berger eigentlich gemeint?" Vorgegeben waren vier Möglichkeiten - von „Er ist sich sicher, dass die Preise bis zum Baubeginn stabil bleiben“ bis $z \mathfrak{u}$ „Er ist sich ziemlich sicher, dass es erhebliche Preissteigerungen geben wird. Hauptsache, der Klient zieht den Auftrag nicht zurück". Die Versuchspersonen, die die negative Fotoserie gesehen hatten, hielten eher die zynische Interpretation für richtig, diejenigen, die die positive gesehen hatten, die ehrliche und aufrichtige.

Man sieht, Bildwirkungen können unter Umständen außerordentlich lang anhalten. Man sieht außerdem, dass die Wirkung dieser Bilder weit über das hinausgeht, was man in den Bildern selbst sehen kann. Die Bilder kreieren Charakterfiktionen, Vorstellungen vom Charakter anderer Menschen, die mit ihrem tatsächlichen Charakter u. U. kaum etwas zu tun haben - es sei denn, Sie unterstellen, jeder hätte mindestens zwei sehr verschiedene Charaktere. Sie werden vielleicht einwenden, das sei ein Laborexperiment und in der Wirklichkeit spiele das keine Rolle. Um das zu prüfen, haben wir Inhaltsanalysen der Fotos u. a. von Reagan und Breschnew im „Stern“ und im „Time Magazine“ gemacht und eine Auswahl der Fotos 200 Testpersonen vorgelegt. Je nachdem, ob die Testpersonen Fotos aus dem „Stern“ oder aus „Time“ gesehen hatten, hielten sie Reagan für inkompetent oder für kompetent bzw. für betrübt oder heiter bzw. für unbeherrscht oder beherrscht. Ähnliche Unterschiede traten in den Urteilen über Breschnew auf. Beide Zeitschriften hatten verschiedene Vorstellungen von der jeweils gleichen Person vermittelt. Bei den Befunden des zuvor berichteten Experimentes handelt es sich demnach nicht um „l'art pour l'art“. Die Bildauswahl spielt in der Realität eine große Rolle, und sie hat faktisch einen eminenten Einfluss auf die Vorstellungen, die wir uns von Personen des öffentlichen Lebens machen. Damit komme ich zu meinem vierten und letzten Punkt.

\section{Viertens: Die Irreführung durch den emotionalen Gehalt von Bildern}

Bilder besitzen kognitive und emotionale Wirkung. Kognitiv meint, sie liefern Informationen über die Wirklichkeit. Wir wissen, jemand hat lange oder kurze Haare, wir sehen, da ist ein Panzer, eine Kirche, eine Ruine usw. Bilder rufen jedoch auch Emotionen hervor, und wenn man Bild- 
Text-Kombinationen analysiert, kann man feststellen: Der Text steuert die Wahrnehmung der Bildinformation, der Bildinhalt verleiht dem Wahrgenommenen Kraft, reichert es mit Emotionen an. Der Bildinhalt ist dafür ausschlaggebend, wie stark Emotionen sind. Der Text ist ausschlaggebend, welche Emotionen ich empfinde, beispielsweise Ärger oder Trauer. Stellen Sie sich folgenden Fall vor: Ein Fußgänger wird von einem Auto angefahren, und diesen Fußgänger sehen Sie auf einem Pressefoto abgebildet. Das Opfer blutet; man erkennt, dieser Mensch leidet. Das Foto vermittelt sozusagen die Power, die Emotion. Aber die Frage bleibt: Welche Emotion? Wenn die Bildunterschrift lautet, der Autofahrer habe alles versucht, um zu bremsen, aber die Bremsen hätten versagt, dann empfinden Sie Trauer. Wenn die Unterzeile aber berichtet, der Autofahrer sei betrunken gewesen und zu schnell gefahren, dann empfinden Sie Ärger, Wut. Diese Emotionen würden Sie vermutlich auch bei einer reinen Textmeldung empfinden. Sie wären aber mit großer Wahrscheinlichkeit längst nicht so stark wie bei der Kombination des Textes mit dem Bild. Der Text gibt die Art der Emotion vor, ihre Stärke beruht auf dem Bild.

Das führt zum Problem der Bildauswahl zurück. Stark emotionalisierende Bilder werden zuweilen in einen textlichen Kontext gestellt, in dem sie sachlich nichts zu suchen haben. Während der BSE-Krise brachte beispielsweise der "Stern“ Ekel erregende Aufnahmen aus Schlachthöfen, Bilder von blutigen Rinderhälften, einem abgehäuteten Rinderkopf usw. Aber was hat es mit BSE zu tun? Ob die Tiere BSE-krank waren oder nicht, konnte der Leser nicht erkennen. Das Ganze hatte keinerlei Informationswert, rief jedoch starke Emotionen hervor, die das Verständnis des Textes überlagerten und seine Wirkung in eine bestimmte Richtung lenkten - gegen „die Fleischindustrie“, ohne die ein Großteil der „Stern“-Leser nur noch selten Fleisch essen könnte.

Nehmen Sie aus jüngster Vergangenheit die SARS-Hysterie: Zu sehen bekam man vermummte Chinesen, die zwar Gefahr signalisierten, aber keinerlei Information über die tatsächliche Gefahr in Deutschland vermittelten. Wir erfuhren weder, ob der Gesichtsschutz gegen die Ansteckung mit SARS half, noch erfuhren wir, wie hoch die Ansteckungsund Sterberate war. Vermittelt wird Angst - nicht Information. Erzielt wird Erregung, nicht Vernunft - und das alles unter dem Deckmantel der Aufklärung und Warnung der Bürger. Als Folge dieses Ausbruchs von Irrationalität verzichteten Zehntausende auf Reisen nach Asien. Die Fluggesellschaften machten Verluste in Milliardenhöhe. Und als dann der Kanzler in dieser Situation nach China reiste, erschien er als wagemutiger Held. Während dessen sind in Deutschland 20000 Menschen unbemerkt an der Grippe gestorben, weil die Medien keine erschreckenden Bilder davon gebracht haben - und der Grippe fiel der gleiche Perso- 
nenkreis zum Opfer, der auch von SARS bedroht war: Alte, Schwache, Vorgeschädigte. Trotzdem ist hier niemand wegen der Grippe-Gefahr mit einem Mundschutz herumgelaufen oder hat sich vor dem Gang in den Supermarkt gefürchtet.

Nehmen Sie als Beispiel die Aufnahmen von den Attacken der Greenpeace-Aktivisten in Schlauchboten auf die Brent Spar, die von Schiffen mit Wasserkanonen abgewehrt wurden. Man bewunderte die mutigen Aktivisten und verurteilte die rücksichtslosen Schiffsbesatzungen. Aber was hatte das mit der Frage $\mathrm{zu}$ tun, ob man die Brent Spar versenken sollte oder nicht? Nichts, es hatte nichts damit zu tun. Nehmen Sie als letztes Beispiel die Aufnahmen von Nematoden im Fisch. Das war Ekel erregend, aber es sagt nichts darüber aus, wie viel Prozent der Fische mit den Würmern belastet waren und ob die Würmer für den Menschen gefährlich oder ungefährlich sind. Selbst wenn einige dieser Beiträge sachlich relevante Informationen enthielten, gingen sie im Abscheu über das Gesehene unter.

Es fehlte in allen genannten Fällen an den wirklich wichtigen Informationen, oder sie wurden allenfalls nebenbei präsentiert. Während der BSE-Krise konnte man in hunderten von Beiträgen im Hitparadenstil erfahren, dass nun das 99., das 100 . und das 101. befallene Rind entdeckt wurde. Die einzig wichtige Information, die man gebraucht hätte, um das Risiko wirklich abschätzen zu können, konnte man dagegen nahezu nirgends finden: den Anteil der erkrankten an allen getesteten Rindern. Hätten die Menschen diese Information gehabt, wäre die hysterische Angst vor BSE vermutlich innerhalb weniger Tage verschwunden: Es waren 0,004 Prozent der getesteten Rinder. Und die Wahrscheinlichkeit, bei normalem Rindfleischgenuss in Deutschland an CreutzfeldtJakob zu erkranken, betrugt im ungünstigsten Fall eins zu fünf Millionen und im günstigsten Fall eins zu 100 Millionen. Zum Vergleich dazu: Die Wahrscheinlichkeit, durch Rauchen an Lungenkrebs zu sterben, ist eins zu 200. Aber Millionen Menschen aßen kein Rindfleisch mehr und trösteten sich mit einer Zigarette.

Bilder haben mehrere Effekte, die auf verschiedenen Ebenen liegen. Sie haben Effekte erster Ordnung - sie wirken auf den Betrachter. Man ist empört, angewidert usw. So wirkten z.B. in der BSE-Krise die erschreckenden Bilder von gequälten Tieren bei Viehtransporten. Solche Transporte sind oft schlimm, haben aber mit BSE nichts zu tun. Die Wirkungen erster Ordnung bestehen in Informationen und vor allem in Emotionen. Die Wirkungen zweiter Ordnung gehen darüber hinaus. Sie erstrecken sich auf das individuelle Verhalten. Man isst kein Rindfleisch, obwohl man sachlich nahezu nichts über die tatsächliche Gefährdung weiß. Die Wirkung dritter Ordnung betrifft die Gesellschaft oder Teile 
von ihr. Der Rindfleischmarkt bricht zusammen, oder der Nudelmarkt oder der Fischmarkt - dies alles haben wir erlebt und Ähnliches steht uns bevor. Das klingt so negativ, aber ich will nicht behaupten, Bilder hätten nur negative Wirkungen.

Ein positives Beispiel für die Wirkung von Bildern ist der Sturz Honneckers und der Zusammenbruch der DDR. Ohne die Bilder der ARD und des ZDF von den Montagsdemonstrationen, die den systemkritischen Menschen in der DDR gezeigt haben, dass sie nicht alleine sind, und ohne die Aufnahmen von den Flüchtlingen aus Ungarn, die ihnen gezeigt haben, dass das Aufbegehren erfolgreich sein kann, wäre die DDR vermutlich nicht kollabiert. Aber als man in der DDR mit eigenen Augen sehen konnte, dass die jungen Leute glücklich und Tränen überströmt über die ungarische Grenze nach Österreich kamen und in Westdeutschland voller Freude aufgenommen wurden, weckte dies starke Emotionen und entsprechend starke Motivationen. Erst dadurch ist aus dem zerstreuten Protest Einzelner jene gewaltige Grundwelle entstanden, die zum Zusammenbruch der DDR geführt hat.

Hierfür gibt es übrigens einen starken Beleg. Wir erinnern uns alle an die Bilder von der Botschaftsbesetzung in Prag, und wir wissen, was das für einen ungeheuren Druck auf die Politik ausgeübt hat. Allerdings war das nicht der erste derartige Fall. Nur wusste das nahezu niemand. Bereits 1984 gab es eine Botschaftsbesetzung, allerdings war es keine Botschaft im rechtlichen Sinn, sondern die westdeutsche Vertretung in 0stBerlin. Dorthin hatten sich über 100 Ostberliner geflüchtet, um ihre Ausreise zu erzwingen. Damals gab es eine Übereinkunft zwischen der Bundesrepublik, der DDR-Regierung und den deutschen Medien, dass darüber nicht berichtet wird, damit es nicht zu einer Eskalation kommt eine berechtigte Furcht. Die Krise wurde gelöst, ohne dass die Menschen hier und in der DDR sie bemerkt hatten. Folglich gab es keine Eskalation. Die DDR hatte noch eine Schonfrist. Ob die Übereinkunft damals berechtigt war, ob man die Fluchtwelle in die westdeutsche Vertretung in OstBerlin verschweigen durfte, lässt sich kaum beurteilen. Mit einiger Gewissheit kann man jedoch sagen, dass die Berichterstattung Folgen gehabt hätte - positive oder negative, das muss offen bleiben.

\section{Ein Fazit}

Bilder besitzen primäre Wirkungen, sie vermitteln Informationen und Emotionen, und sie haben sekundäre Wirkungen, Wirkungen zweiter Ordnung, sie verändern unser Verhalten. Und dieses veränderte Verhalten wird unter Umständen selbst zum Gegenstand der Berichterstattung. 
Schließlich können die durch Bilder hervorgerufenen Verhaltensänderungen als Wirkungen dritter Ordnung erhebliche gesellschaftliche Konsequenzen besitzen. Ich werde Ihnen gleich noch ein Beispiel in Erinnerung rufen, das Sie kennen.

Das Problem der Verfälschung von Bildern besteht nicht darin, dass die vorhandenen Bilder verfälscht werden. Das Problem der verfälschenden Bildberichterstattung besteht schon darin, dass die Bilder etwas zeigen, was es ohne die Kameras gar nicht gäbe. Das ist sogar das Hauptproblem. Der Zuschauer hat in der Regel keine Chance festzustellen, wie die Existenz der Kameras, der Beleuchtungseinrichtungen und der Aufnahmeteams das Verhalten der abgebildeten Menschen verändert. Jeder, der Gelegenheit hat, einen prominenten Politiker im engeren Kreis zu treffen, wird feststellen, dass er ganz anders ist als er im Fernsehen erscheint. Manche wirken im Fernsehen hölzern und erweisen sich im kleinen Kreis als charmant und geistesgegenwärtig. Andere sind dagegen im direkten Kontakt keineswegs so locker und witzig wie sie im Fernsehen erscheinen. Mein letztes Thema war die Irreführung durch bildlich ausgelöste Emotionen. Bilder können emotionale Reaktionen hervorrufen, die unter Umständen sachlich völlig inadäquat sind. Sie wecken übertriebene Ängste oder Hoffnungen, die individuelle Verhaltensänderungen hervorrufen. Sie können wiederum erhebliche gesellschaftliche Konsequenzen besitzen, die man - je nach Standpunkt und Interessenlage - positiv oder negativ bewerten mag. Nur bedeutungslos sind sie nicht.

Als Abschluss ein Beispiel für eine Wirkung dritter Ordnung. Ein Beispiel dafür, wie die Bilder die Wirklichkeit verändern. Sie erinnern sich an die Somalia-Intervention der USA. Vorangegangen war eine Kampagne der amerikanischen Fernsehsender, die wahrheitsgemäß und Mitleid erregend das Elend in Somalia und seine Ursachen angeprangert hatten. Zugleich vermittelten sie den Eindruck, die USA - ja die Weltgemeinschaft insgesamt - müssten militärisch intervenieren, um den Banden ein Ende zu bereiten, die so viel Elend über die Bevölkerung gebracht hatten. Dies erzeugte einen ungeheuren Druck vor allem auf die amerikanische Regierung. Der damalige Präsident Bush hat diesem Druck wohl auch mit Blick auf die bevorstehenden Präsidentschaftswahlen nachgegeben. Als die Operation anlief, waren zum ersten Mal in der Kriegsgeschichte die Kamerateams schussbereit schon vor den Soldaten am Einsatzort, um die ankommenden Landungstruppen zu filmen. Die hollywoodreife Situation am "set“ wurde schnell zum Gegenstand kritischer Kommentare in anderen Medien, wobei allerdings nicht recht deutlich wurde, dass die Truppen ohne die früheren Fernsehberichte aller Wahrscheinlichkeit nach gar nicht erst dort gelandet wären. Es ging im Kern 
nicht darum, dass sich die Kamerateams distanzlos in das Geschehen eingegliedert hatten - es hätte ohne die frühere Berichterstattung gar nicht stattgefunden. Als amerikanische Soldaten erschossen und ein toter Amerikaner vor den Fernsehzuschauern durch Mogadischu geschleift worden war, löste dies vor allem in den Medien der USA eine Welle der Empörung aus, die in die medial verstärkte Forderung mündete: „Bring our boys back!" Erneut folgte die Politik den Forderungen der Medien vor allem des Fernsehens, das sie erst in die missliche Lage gebracht hatte. Die Amerikaner und ihre Verbündeten zogen unter dem Druck vor allem des Fernsehens überhastet aus Somalia ab und ließen die Bevölkerung mit den gewalttätigen Banden mehr oder weniger so zurück, wie sie sie vorgefunden hatten.

Das Ergebnis der ersten Berichte war ein gewaltsamer Konflikt, der ein paar hundert, vielleicht auch ein paar tausend Tote gekostet, aber Elend nicht beseitigt hat. Bleibt die Frage nach der Verantwortung: Wer hat eigentlich die Verantwortung für die missglückte Intervention? Liegt sie bei den Fernsehsendern NBC, CBS und ABC, oder liegt sie bei der Regierung Bush, oder bei beiden, oder bei den Zuschauern, die sich $\mathrm{zu}$ emotional verständlichen, sachlich aber kaum vertretbaren Interventionsforderungen haben hinreißen lassen? Auf jeden Fall kann man mit einiger Sicherheit sagen: Diese ganze Intervention hätte nie stattgefunden, und die Konsequenzen wären nie eingetreten, wenn es die Bilder vom Elend nicht gegeben hätte und die Bilder von den toten Amerikanern. Die Bilder waren nicht gefälscht. Niemand hat - soweit wir wissen Aufnahmen manipuliert. Alles wurde so gezeigt, wie es im Moment der Aufnahme war. Aber ohne die Aufnahmen wäre vieles nicht so gewesen, wie es dann gezeigt wurde. Es wurde ein greller Scheinwerfer auf irgendeine Ecke des Elends der Welt gelegt, und andere Ecken wurden nicht beleuchtet. Und weil wir keine Bilder von ihrem Elend gesehen haben, haben sie unser Mitleid nicht erregt, und die Amerikaner und ihre Verbündeten waren nicht da, um sie von ihrem Elend zu erlösen, und man kann vielleicht sagen, sie haben Glück gehabt. 